Western University Scholarship@Western

1977

\title{
Costly Innovation and Natural Resources
}

Arthur J. Robson

Follow this and additional works at: https://ir.lib.uwo.ca/economicsresrpt

Part of the Economics Commons

Citation of this paper:

Robson, Arthur J.. "Costly Innovation and Natural Resources." Department of Economics Research Reports, 7707. London, ON: Department of Economics, University of Western Ontario (1977). 
RESEARCH REPORT 7707

COSTLY INNOVATION AND NATURAL RESOURCES

by

Arthur J. Robson

\begin{abstract}
An optimal model explores the connection between costly innovation and resource depletion. Both phenomena affect the asymptotic growth rate, whereas capital accumulation does not. However, there is not a simple trade-off between these two phenomena. Less productive innovation can mean a faster rate of exploitation of the depletable resource. A larger output share for the depletable resource can mean less innovation and faster exploitation. The Rawlsian criterion is treated as a limiting case. Then the rent from the depletable resource could finance ipnovation, and no investment should occur. The paradoxes above make the Rawlsian position seem less extreme.
\end{abstract}




\section{Introduction}

The realization that land is in fixed supply led Malthus to the gloomy prediction of perpetual long-run subsistence. In contrast, neoclassical economics has tended to stress the role of capital in promoting growth. Hotelling [5] was among the first to bring neoclassical analysis to bear on the question of limited (indeed depletable) natural resources. In particular, he studied the influence of market structure. A more recent upsurge of interest in natural resources seems to have been sparked by the oil crisis and the apocalyptic vision of the book The Limits to Growth (Meadows, et al. [7]). Much of the recent work has focussed on the substitution in production of capital for the natural resource. This possibility alone can vitiate the pessimistic Limits to Growth conclusions (see Solow [11], for example). A potent force besides investment leading to optimistic conclusions is innovation. There can be no doubt as to the historical importance of innovation (as distinct from capital augmentation) in promoting growth. A problem is that innovation is less susceptible to precise modelling (as a result of its very nature). In the context of earlier neoclassical growth there is a substantial literature on exogenous innovation (see Arrow and Kurz [1] for a typical model). "Endogenous" innovation is usually taken to refer to choice of the factor bias. (See Kennedy [6].) However, Phelps [8] for example, considers costly innovation. In the recent context of natural resources, costly innovation has been studied by Dasgupta and Heal [4]. In their article technological change is modelled as a quantum jump--a breeder or fusion reactor ushers in the energy millenium. (Natural resources may become useless, in fact.) The date of the innovation 
Is random (although advanced by expenditure on research). However, the properties of the innovation are precisely known.

The present paper models costly but continuous innovation. While quantum jumps may occur in terms of inventions, the overall process must be much smoother when development is also considered. Indeed, perhaps the supply of inventions is quite inelastic with respect to expenditure; however, this is less likely in the case of development. Two types of limited natural resources are considered in the paper. These types are exemplified by oil on the one hand and by hydroelectricity (or land) on the other. The first type then has a fixed initial stock and is depletable. The second type is in fixed flow supply--although it is nondepletable it is equally non-augmentable.

The time-honored method of modelling myopia in growth models is to assume a constant savings rate. The counterparts of this assumption needed to close the present model are taken to be (1) A constant fraction of output is used to finance innovation. (2) The "rate of exploitation" of the depletable resource is constant. (This is the ratio of the resource flow to the current stock.) It is shown that the fraction of output used to finance innovation and the rate of exploitation of the depletable resource are more fundamental than the savings rate since they affect the endogenous (asymptotic) growth rate.

Under a fully optimal regime it is shown that an appropriate constant savings rate and appropriate choices under (1) and (2) above are asymptotically obtained. In the course of discussion of the optimal model, economic interpretations of the efficiency conditions are noted. In particular, the appropriate rate of return on innovation is derived. 
Optimal paths are shown to converge to balanced growth (or "decay") paths. Comparative long-run "statics" show that effects on endogenous rates of growth replace more familiar effects in terms of percentage shifts in the paths of varlables of interest, with a fixed growth rate.

Paradoxically, it is shown that more productive innovation might lead to a faster rate of exploitation of the depletable resource. Again it is possible that a larger relative share of the depletable resource might call for less Innovation and a faster rate of exploitation of the depletable resource.

The Rawlsian criterion can be treated as a limiting case. Efficlency dictates that the stocks of capital, technology, and the depletable resource be generally readjusted. The state of the economy is not then immediately "frozen" as with capital only. (See Solow [11].) It 1s shown that eventually rent from the depletable resource should equal the cost of innovation, whereas the stock of capital should be fixed. Furthermore, the rate of exploitation of the depletable resource ultimately equals the asymptotic rate of interest. 


\section{The Mode1}

Consider firstly the production function. This is taken to be CobbDouglas. Although analytic feasibility dictates this cholce to a large extent, the Cobb-Douglas case captures the essence of the problem with a depletable resource. (The resource is essential, in the sense that output is zero without it. Also the marginal product of the resource tends to infinity as the resource input tends to zero. Somewhat surprisingly, these two stipulations leave only the Cobb-Douglas case among the CES class. See Dasgupta and Heal [4].) Hence output is taken to be

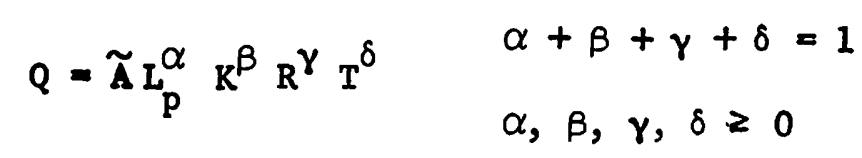

where $\tilde{A}$ is an efficiency parameter (which reflects innovation), $L_{p}$ is labor used in production (the remainder being used for innovation), $K$ is the capital stock, $R$ is the flow of the depletable resource used up in production, and $T$ is the stock of the non-depletable resource. For the sake of economy of potation redefine the efficiency parameter to include the non-depletable resource. Then (1) becomes

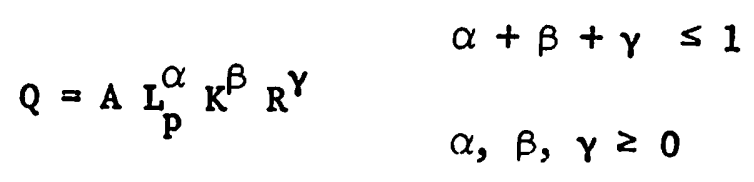

(Note for future reference--see Proposition 5--that changing the value of $\gamma$ while holding $\alpha$ and $\beta$ constant corresponds to altering the relative shares of the depletable and non-depletable resources. The polar case with only a depletable resource corresponds to $\gamma=1-\alpha-\beta$; the case with only a nondepletable resource to $\gamma=0$.) 
Suppose that the stock of the depletable resource at time $t$ is $S(t)$ and that there is a given Initial stock of $s_{0}$, then

$$
s(t)=\int_{t}^{\infty} R(t) d t, \quad s(0)=s_{0}
$$

where

$$
\epsilon=\frac{R(t)}{S(t)}=-\frac{S^{\prime}(t)}{S(t)}
$$

is the "rate of exploitation."

Although the production function, (2), exhibits increasing returns to scale when $A$ is treated as a factor, this is later shown not to cause a problem given the specification of innovation. In fact, innovation Is taken to be determined by

$$
\frac{A^{\prime}}{A}=\frac{e L_{I}}{L} \text { where } L=L_{p}+L_{I}
$$

where $L_{I}$ is the labor used for innovation, $L$ is the total labor force, and e is a positive parameter. The labor force is taken to be constant. Exponential growth would not cause substantial complications but it is perhaps unreasonable in the present context if only for reasons of standing room.

Consider now the interpretation of (4). With a more general production function, and an essential depletable resource, it seems that depletable-resource-augmenting technological change would be eventually needed to stave-off collapse. However, in the Cobb-Douglas case, Hicks-neutral and factor augmenting technological change are equivalent. Hence (4) can be taken to mean that the growth in effective labor is proportional to the labor employed in innovation. The homogeneity of (4) in $\mathrm{L}_{I}$ and $\mathrm{L}$ means that 
optimal innovation is independent of the scale of the entire economy (or of each firm within an economy). The linearity of (4) in $I_{I}$ is an assumption of constant returns in innovation for a given total labor force. An important point about (4), in the present context, is that it permits endogenous constant asymptotic growth. (As in Conlisk [3].)

Finally, since there is one (tangible) output,

$$
K^{\prime}=Q-C
$$

where $C$ is consumption. Depreciation of capital can be handled by notational changes but is omitted for the sake of simplicity. (See Arrow and Kurz [1].) 3. Myopic Evolution Over Time

Consider the outcome with a constant savings rate, a constant rate of innovation, and a constant rate of exploitation of the depletable resource. Particular constant values of these parameters will be shown to be asymptotically optimal.

\section{Proposition 1}

A constant rate of innovation arises if a constant fraction of output, $f$, is used to finance innovation. As long as the rate of exploitation of the depletable resource, $\varepsilon$, is not too large, the capital stock, $K$, output, Q, and consumption, $C$, grow asymptotically at the same positive rate. This growth rate is independent of the savings rate, $s$, but does depend on $\varepsilon$ and $f$.

Proof

Since the value of labour used for innovation is $Q_{L} \cdot L_{I}$,

$$
f Q=Q_{L} \cdot L_{I}=Q_{L} \cdot\left(L-L_{P}\right)
$$


which Implies the fraction of labor used in production, $x$, is given by

$$
x=\frac{I_{p}}{L}=\frac{\alpha}{\alpha+f}, \quad 0<x \leq 1
$$

Now

$$
\frac{A^{\prime}}{A}=\frac{e L_{I}}{L}=e(1-x)=\frac{e f}{\alpha+f}
$$

so that there is a constant rate of innovation. Now if the rate of exploitation is a constant, $\varepsilon$,

$$
R=R_{0} \exp (-\epsilon t)
$$

Hence

$$
K^{\prime}=s Q=s \Lambda_{0} \exp \left[\frac{e f t}{\alpha+f}\right] x^{\alpha} L_{K} \beta_{R_{0}} \gamma \exp [-\gamma \in t]
$$

As suming that

$$
\text { ef } /(\alpha+f)>\gamma \epsilon
$$

integration yields

$$
\mathrm{K}^{1-\beta}-\mathrm{K}_{0}^{1-\beta}=\mathrm{C}\left\{\exp \left[\operatorname{ef} /(\alpha+f)-\gamma^{\varepsilon}\right] \mathrm{t}-1\right\}
$$

where $C$ is a positive constant. Hence, asymptotically,

$$
\frac{K^{\prime}}{K}=[e f /(\alpha+f)-\gamma \varepsilon] /(1-\beta)
$$

Now

$$
\begin{aligned}
\frac{C^{\prime}}{C} & =\frac{Q^{\prime}}{Q}=\frac{A^{\prime}}{A}+\beta \frac{K^{\prime}}{K}+\gamma \frac{R^{\prime}}{R} \\
& =[e f /(\alpha+f)-\gamma \varepsilon] /(1-\beta)
\end{aligned}
$$


Note 1: If there is only a non-depletable resource (if $\gamma=0$ ) then (11) Is satisfied for $\mathrm{f}>0$.

Note 2: Under the optimal regime to be discussed next, it is possible for "balanced decay" to occur. Constant values of $s, \epsilon$, and $f$ obtain asymptotically. However, there is a relationship between these values, and the analogous general result to that presented in Proposition 1 is not true.

Note 3: Stiglitz [12], studies a similar model with exogenous innovation. He obtains the result that the rate of savings, 8 , influences the asymptotic growth rate. However, he assumes an efficiency condition between the depletable resource and capital. Then a different savings rate and hence a different rate of return on capital imply a different rate of exploitation of the depletable resource. Stiglitz's result is thus consistent with Proposition 1.

The present model is more general since it does not rely on the existence of a depletable resource to induce an endogenous asymptotic growth rate.

4. Optimality Conditions

Consider now an optimum allocation. Take the dynamic utility functional to be, for the sake of simplicity,

$$
u[c]=\int_{0}^{\infty} \frac{c^{1-\sigma}}{1-\sigma} e^{-\rho t} d t
$$

where $\rho$ is the explicit rate of time preference, and $\sigma$ is the elasticity of marginal utility with respect to consumption, $C$. (Take $\sigma>0, \sigma \neq 1$. Instead of $\sigma=1$, take the logarithmic case.) The optimum is found by maximizing (15) subject to the differential equations (4) and (5) for the efficiency parameter, A, and capital, K, respectively, and subject also to the constraint (3) on use of the depletable resource. (Initial values must be given for $\mathrm{A}$ and $\mathrm{K}$ also.) The appropriate present-valued Hamiltonian is 


$$
\mathcal{K}=e^{-\rho t}\left\{\frac{C^{1-\sigma}}{1-\sigma}+\Psi \frac{e\left(L-L_{p}\right)}{L} A+\phi\left(A L_{p}^{\alpha} K^{\beta} R^{\gamma}-C\right)\right\}-\lambda R
$$

which is to be maximized over choice of consumption, C, labor used in production, $L_{p}$, and use of the depletable resource, $R$. The variables $\Psi$ and $\phi$ are current shadow prices of $A$ and $K$, respectively. The multiplier $\lambda$ is the constant present-value shadow price of the depletable resource. (Hence the current shadow price of the depletable resource rises exponentially at rate $\rho$ as in Hotelling [5].) Assuming an interior solution (this can be justified),

$$
\frac{\partial T C}{\partial C}=0 \text { hence } C^{-\sigma}=\varnothing
$$

equating the marginal instantaneous utility of consumption to the current price of capital, as expected in a one good world. Also,

$$
\frac{\partial \digamma C}{\partial R}=0 \text { or } \phi Q_{R}=\gamma \phi A L_{p}^{\alpha} K_{R}^{\beta} R^{\gamma-1}=\lambda e^{\rho t}
$$

equating the current marginal value product of the depletable resource to its current price. Both (17) and (18) could arise from (idealized) competition as usual. Finally,

$$
\frac{\partial T C}{\partial L_{p}}=0 \text { hence } \frac{\Psi \text { e A }}{L}=\phi \alpha A L_{p}^{\alpha-1} \mathrm{~K}^{\beta} \mathrm{R}^{\gamma}
$$

Clearly this rule optimally allocates a given labor force, $L$, between Its two alternative uses--for production or for innovation. Although the shadow price $\Psi$ cannot be readily interpreted as a competitive price, to decentralize the optimum it suffices to assume a firm undertakes innovation on its own behalf. It then retains an internal shadow price for innovation. No diffusion of innovation is necessary. For it can be shown that each competitive firm (a scaled-down replica of the entire economy) will employ the same optimal fraction of its labor force for innovation. 
Consider now the adjoint equation for $\phi$, the price of capital (or consumption from (17)). As usual,

$$
\frac{\phi^{\prime}}{\phi}=\rho-Q_{K}=\rho-\beta y,
$$

where

$$
y=Q / K
$$

is thus the output-capital ratio. From (17), (20) is equivalent to

$$
Q_{K}=\rho+\sigma \frac{C^{\prime}}{C}=r_{c}
$$

where $r_{c}$ is the consumption rate of interest. Equation (21) has the usual Fisherian interpretation of equating the subjective and objective rates of interest. Also, from (18) and (20),

$$
\frac{Q_{R}^{\prime}}{Q_{R}}=Q_{K}=B y
$$

which equates the return on holding the depletable resource (a capital gain) to the return on capital (its marginal product). This is an efficiency condition.

Consider now the adjoint equation for (4), the shadow current price of the technology parameter, A,

$$
\Psi^{\prime}-\rho \Psi=-\frac{\Psi e\left(L-L_{p}\right)}{L} A-\phi L_{p}^{\alpha} K^{\beta} R^{\gamma}
$$

which can be rewritten, using (19), as

$$
\frac{\Psi^{\prime}}{\Psi^{\prime}}=\rho-\mathrm{e}\left(1+\frac{1-\alpha}{\alpha} \mathrm{x}\right), \quad \mathrm{x}=\mathrm{L}_{\mathrm{p}} / \mathrm{L}
$$

as before. Consider now the efficiency condition between innovation and capital. Differentiating (19) logarithmically, and using (20) and (24),

$$
\frac{e x}{\alpha}+\frac{Q_{L}^{\prime}}{Q_{L}}=Q_{K}=\beta y
$$


Note that since this equation depends only on relative factor intensities, that decentralization of the optimum is possible as was asserted following (4) and (19).

To interpret the left-hand side of (25), consider the rate of return on the wages of labor used for innovation during a short time, $6 t$. The wage bill is

$$
Q_{L} L_{I} \delta t=\left(\alpha Q / L_{p}\right) L_{I} \delta t
$$

On the other hand, the increase in output due to innovation is

$$
(\delta A / A) Q=\left(A^{\prime} / A\right) Q \delta t=\left(e L_{I} / L\right) Q 6 t
$$

so a natural definition of the rate of return on innovation is :

$$
r_{I}=\frac{\left(e L_{I} / L\right) Q \delta t}{\left(\alpha Q / L_{p}\right) L_{I} \delta t}=\frac{e L_{p}}{\alpha I}=\frac{e x}{\alpha}
$$

which is the first term in (25). To interpret the second term, consider the labor hired now for time $\delta$ rather than in the immediate future for time $\delta t$. The rate of return on this variation is the second term. From (25), if the wage rate is rising, the rate of return on innovation ( $r_{I}$ as in (28)) should be driven below the marginal product of capital, because it pays to innovate now using cheaper labor rather than later. Conversely, if the wage rate is falling, innovation should be postponed, in the sense that its rate of return should exceed the marginal product of capital. This result is formalized in the following.

\section{Proposition 2}

The efficiency condition relating innovation and capital, (25), states that the sum of the rate of return on innovation and the rate of increase in the wage rate should equal the marginal product of capital. 


\section{Optimum Evolution Over Time}

Consider now the evolution of the optimal path over time. Note this problem involves three state variables--capital, $K$, technology, $A$, and the stock of the depletable resource, S. Such problems cannot generally be "solved" without extra assumptions. (Typically including a "small" rate of explicit time preference. See Brock and Scheinkman [2], for example.) However, this problem needs no such extra assumptions. Firstly, consider the definitions

$$
x=\frac{L_{p}}{L}, \quad y=\frac{Q}{K}, \quad z=\frac{C}{K}, \quad 0<x \leq 1
$$

where $x$ and $y$ are as already introduced. The "equations of motion", (5) and (6), become

$$
\frac{A^{\prime}}{A}=\frac{e L_{I}}{L}=e(1-x)
$$

and

$$
\frac{\mathrm{K}^{\prime}}{\mathrm{K}}=\frac{\mathrm{Q}}{\mathrm{K}}-\frac{\mathrm{C}}{\mathrm{K}}=\mathrm{y}-\mathrm{z}
$$

respectively. The efficiency conditions are (22) and (25). After some algebra, these can be shown to imply

$$
(1-\alpha-\gamma) \frac{x^{\prime}}{x}=e\left\{1+\frac{1-\alpha-\gamma}{\alpha} x\right\}-\beta z
$$

and

$$
(1-\alpha-\gamma) \frac{R^{\prime}}{R}=e-\beta z
$$

Consider the behavior of $y$, the output-capital ratio, along an optimal path. Since

$$
\frac{\mathrm{y}^{\prime}}{\mathrm{y}}=\frac{\mathrm{Q}^{\prime}}{\mathrm{Q}}-\frac{\mathrm{K}^{\prime}}{\mathrm{K}}=\frac{\mathrm{A}^{\prime}}{\mathrm{A}}+\alpha \frac{\mathrm{L}_{\mathrm{P}}^{\prime}}{\mathrm{L}_{\mathrm{p}}}+\beta \frac{\mathrm{K}^{\prime}}{\mathrm{K}}+\gamma \frac{\mathrm{R}^{\prime}}{\mathrm{R}}-\frac{\mathrm{K}^{\prime}}{\mathrm{K}}
$$

it can be shown, using (29) through (33), that

$$
(1-\alpha-\gamma) \frac{y^{\prime}}{y}=e-(1-\beta)(1-\alpha-\gamma) y+(1-\alpha-\beta-\gamma) z
$$

Using the optimality condition (21), 


$$
\sigma \frac{z^{\prime}}{z}=\sigma z-(\sigma-\beta) y-\rho
$$

Note now that the differential equations for $y$ and $z,(35)$ and (36), are self-contained, in the sense that no other variables appear in these equations. This facilitates a (generalized) phase-diagram approach. Represent (35) and (36) in the $y-z$ plane, as in Figure 1. The slope of $z^{\prime}=0$ locus can be easily shown to exceed algebraically that of the $y^{\prime}=0$ locus. The unique equilibrium in the $y-z$ plane is

$$
\begin{aligned}
& y^{*}=\frac{\sigma e+(1-\alpha-\beta-\gamma) \rho}{\beta[(1-\beta)-(\alpha+\gamma)(1-\sigma)]} \\
& z^{*}=\frac{(\sigma-\beta) e+\rho(1-\beta)(1-\alpha-\gamma)}{\beta[(1-\beta)-(\alpha+\gamma)(1-\sigma)]}
\end{aligned}
$$

For each initial value of $y$, there is a unique initial value of $z$, such that the path satisfying (35) and (36) converges monotonically to the equilibrium (37). (An analytic proof of this is not difficult.) This path can be shown to be optimal by application of a sufficiency theorem.

Intuitively, however, what is wrong with other paths? Clearly other paths either cross $z=0$ in finite time or have $z$ tending to infinity. Those with $z=0$ in finite time have then zero consumption but a positive capital stock, K. This is non-optimal. Those with $z$ tending to infinity exhaust the capital stock in finite time and are infeasible.

Suppose, therefore, that $z$ (and $y$ ) follow the path converging to $z^{*}$ (and $y^{*}$ ). Then the path of $x$ can be deduced from (32). The situation is represented in the $x-z$ plane in Figure 2 . It is readily shown that a unique equilibrium value of $x$, is given by 


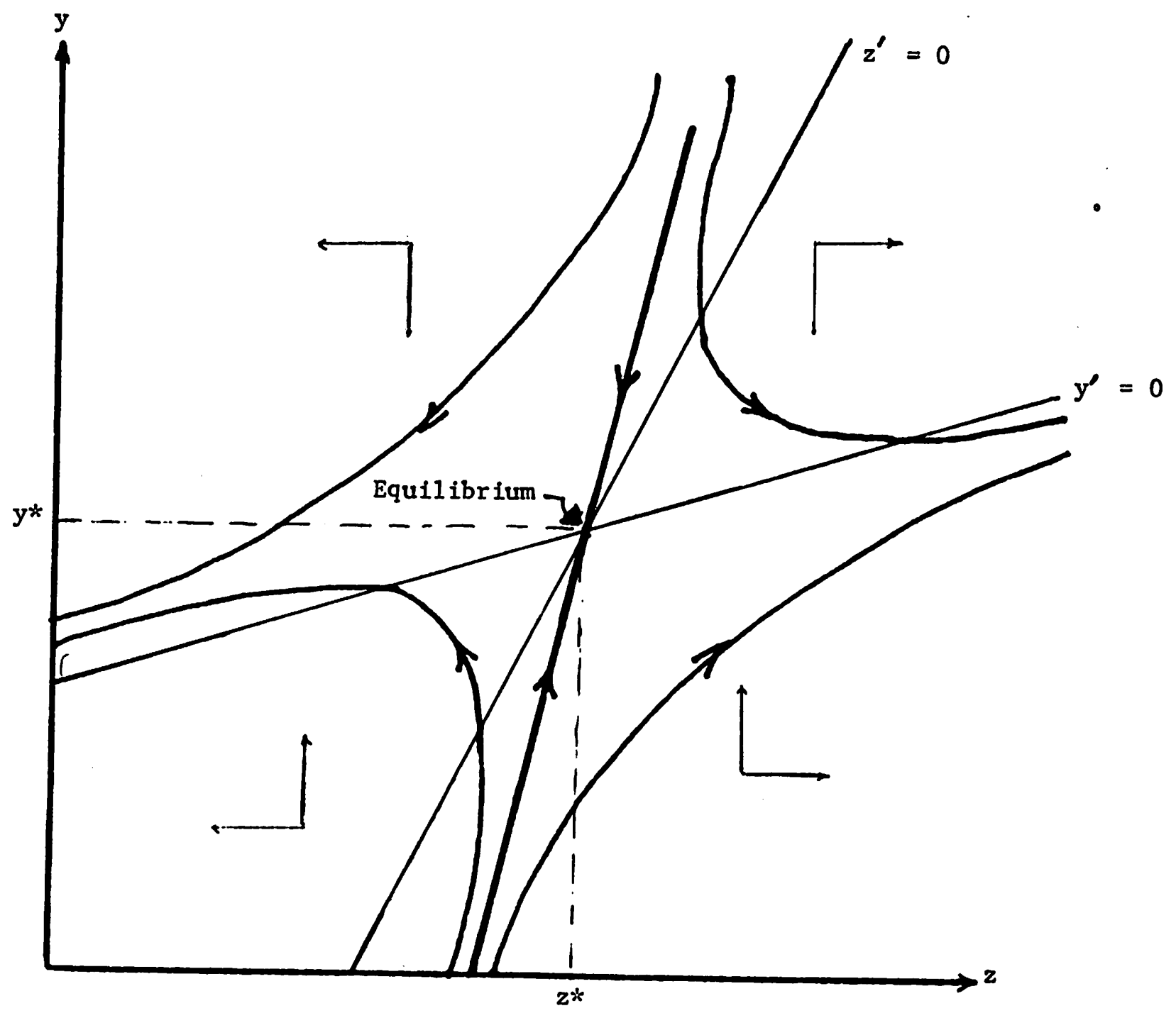

Figure 1 


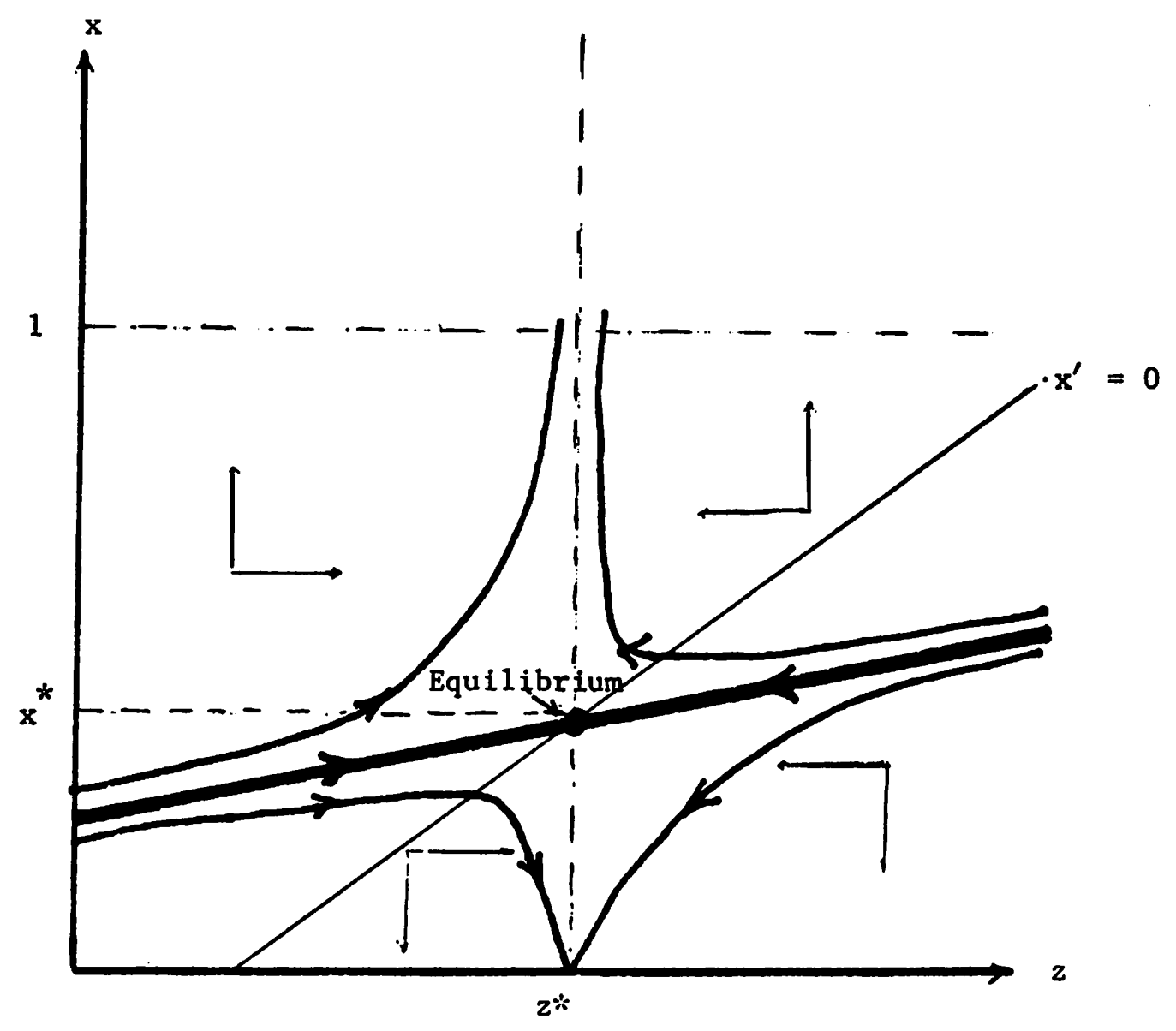

Figure 2 


$$
x^{*}=\frac{\alpha[\rho(1-\beta)-e(1-\sigma)]}{e[(1-\beta)-(\alpha+\gamma)(1-\sigma)]}
$$

In order to obtain $0<x^{*}<1$ (and incidentally, $z^{*}>0$ ), the following conditions are assumed

$$
e(1-\sigma)<\rho(1-\beta)
$$

and

$$
\alpha(1-\beta) \rho<e[(1-\beta)-\gamma(1-\sigma)]
$$

Note (39) is satisfied, in particular, for large $\sigma$, the elasticity of marginal utility. Clearly, from Figure 2, to each initial value of $z$, there corresponds a unique initial value of $x$, such that $x$ converges monotonically to $x^{*}$. This path will be shown to be optimal by means of the sufficiency theorem. Paths which start too high become infeasible by crossing $x=1$. Paths starting too low involve an unnecessarily low consumption stream.

Now given an initial value of $x$, say, the time paths of all variables of interest follow by the requirement that the convergent path be followed. Hence the paths of $x, y$, and $z$ are determined. Equations (30) and (31), together with initial values, determine the paths of $\mathrm{A}$ and $\mathrm{K}$ respectively. Given $y, z$ and $K$ the paths of $C$ and $Q$ follow. Then the path of $z$ and the initial stock of the depletable resource determine the path of $R$, from (33). Note that

$$
\begin{aligned}
\varepsilon^{*} & =\lim _{t \rightarrow \infty} \frac{R}{S}=\lim _{t \rightarrow \infty}-\frac{R^{\prime}}{R} \\
& =\frac{\beta z^{*}-e}{1-\alpha-\gamma}=\frac{\rho(1-\beta)-e(1-\sigma)}{[(1-\beta)-(\alpha+\gamma)(1-\sigma)]}>0
\end{aligned}
$$


under (39), so that the integral (3) converges. Finally, the asymptotic rate of growth follows from (31), it is

$$
w^{*}=y^{*}-z^{*}=\frac{e-\rho(\alpha+\gamma)}{[(1-\beta)-(\alpha+\gamma)(1-\sigma)]}
$$

which can be positive or negative, depending on whether

$$
\text { e }<\rho(\alpha+\gamma)
$$

which is a condition independent of (39) since it does not involve $\sigma$. How then is the initial value of $x, x_{0}$, determined? Note that the use of the depletable resource at $t=0$ depends only on $x_{0}$. In fact, it can be shown that

$$
\mathrm{dR}_{\mathrm{o}} / \mathrm{dx} \mathrm{x}_{\mathrm{o}}>0
$$

Now, at $t=0$

$$
y_{0}=A_{0} x_{0}^{\alpha} L^{\alpha} K_{0}^{\beta-1} R_{0}\left(x_{0}\right)^{\gamma}=I_{0}\left(x_{0}\right)
$$

say. This relationship determines $x_{0}$. The situation is represented in Figure 3. Existence of suitable $x_{0}$ between 0 and 1 is simply assumed. Uniqueness is a consequence of the sufficiency theorem. Figure 3 also depicts the equilibrium situation with

$$
y^{*}=\left(\mathrm{A} \mathrm{K}^{\beta-1} \mathrm{R}^{\gamma}\right)^{*}{ }_{\mathrm{x} * \alpha}{ }_{\mathrm{L}}^{\alpha}=\mathrm{I} *\left(\mathrm{x}^{*}\right)
$$

say, where although the individual terms in parentheses are not constant, their product is. (Equations (42) and (43) are again independent of scale.) If the economy is initially "capital poor" then

$$
y *<I_{0}(x *)
$$




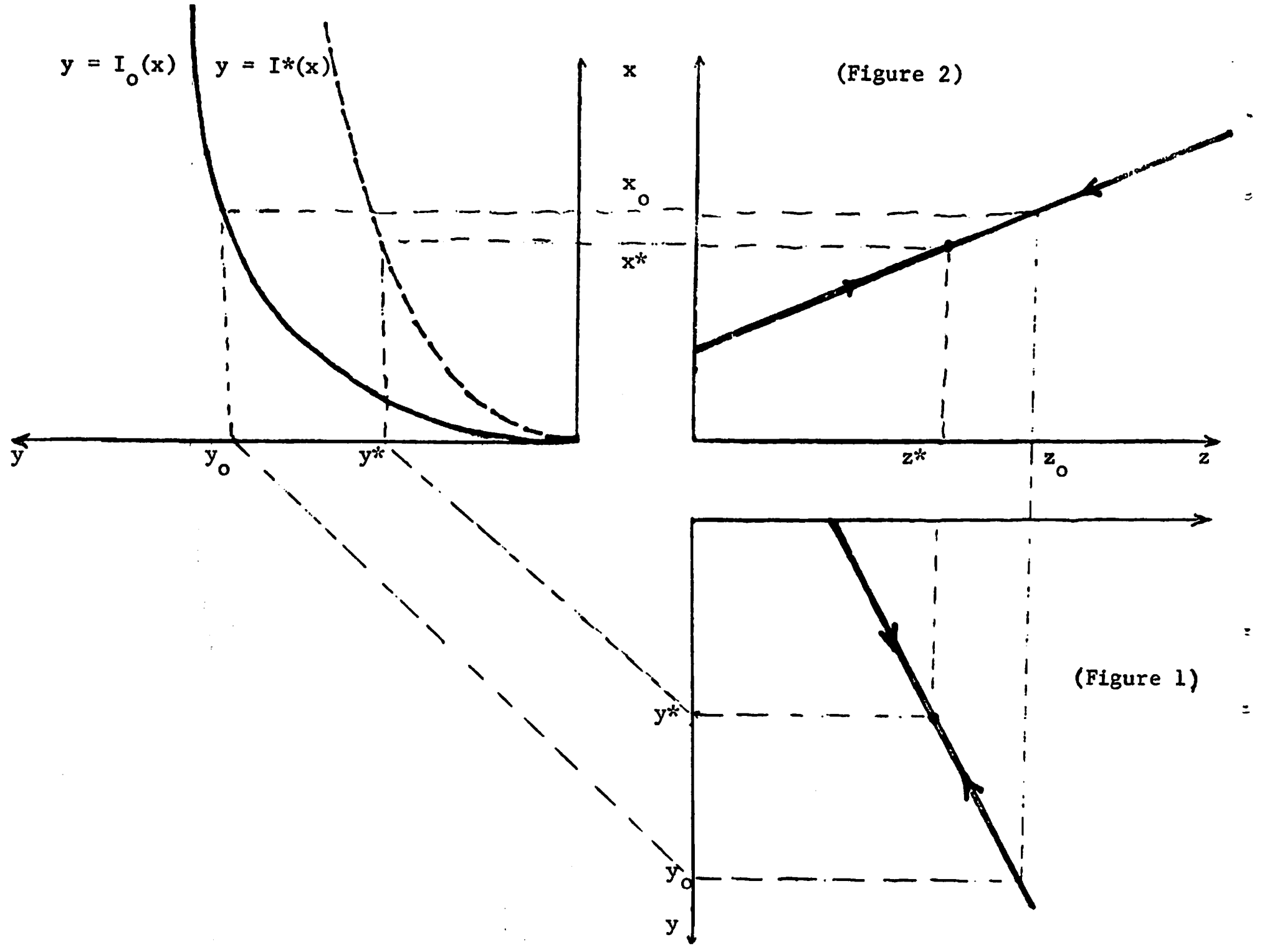

Figure 3 
and Figure 3 suggests that $x, y$, and $z$ decline monotonically to their equilibrium values. The converse would be that an initially "resource poor" ecopomy should follow a path with $x, y$, and $z$ rising monotonically to their equilibrium values. However, general results concerning the direction of convergence seem difficult to obtain.

The following theorem restates compactly the hypotheses and main conclusions of the foregoing analysis. The applicability of the sufficiency theorem is demonstrated. Subsequent propositions investigate the comparative long-run "statics" of the balanced growth path.

Theorem. Consider the model of costly technological change and natural resources defined by

$$
\begin{aligned}
& Q=\tilde{A} L_{P}^{\alpha} K^{\beta} R^{\gamma} T^{\delta}=A L_{P}^{\alpha} K^{\beta} R^{\gamma} \quad \alpha+\beta+\gamma \leq 1 \\
& \int_{0}^{\infty} R d t=S \\
& A^{\prime} / A=e L_{I} / L, \quad I=L_{p}+L_{I} \\
& K^{\prime}=Q-C \\
& u[C]=i_{0}^{\infty} \frac{C^{1-\sigma}}{1-\sigma} e^{-\rho t} d t
\end{aligned}
$$

Then, the optimal path exists, is unique and involves perpetual innovation if conditions (39) hold. Indeed the optimal path is such that

$$
x=L_{p} / I, \quad y=Q / K, \quad \text { and } z=C / K
$$

tend monotonically to equilibrium values 


$$
\begin{aligned}
& x^{*}=\frac{\alpha[\rho(1-\beta)-e(1-\gamma)]}{e[(1-\beta)-(\alpha+\gamma)(1-\sigma)]} \\
& y^{*}=\frac{\sigma e+(1-\alpha-\beta-\gamma) \rho}{\beta[(1-\beta)-(\alpha+\gamma)(1-\sigma)]} \\
& z^{*}=\frac{(\sigma-\beta) e+\rho(1-\beta)(1-\alpha-\gamma)}{\beta[(1-\beta)-(\alpha+\gamma)(1-\sigma)]}
\end{aligned}
$$

The common asymptotic growth rate of $\mathrm{K}, \mathrm{Q}$, and $\mathrm{C}$ is given by

$$
w^{*}=\frac{e-\rho(\alpha+\gamma)}{[(1-\beta)-(\alpha+\gamma)(1-\sigma)]}
$$

which may be either positive or negative. The asymptotic rate of depletion of the depletable resource is

$$
\varepsilon^{* .}=\lim _{t \rightarrow \infty} \frac{R}{S}=\frac{\rho(1-\beta)-e(1-\sigma)}{[(1-\beta)-(\alpha+\gamma)(1-\sigma)]}
$$

The asymptotic savings rate is

$$
\mathbf{s}^{*}=\left(\frac{\mathrm{K}^{\prime}}{Q}\right)^{*}=\frac{\mathrm{y}^{*}-\mathrm{z}^{*}}{\mathrm{y}^{*}}=\frac{\mathrm{e}-(\alpha+\gamma) \rho}{\sigma \mathrm{e}+(1-\alpha-\beta-\gamma)}
$$

and the fraction of output needed to finance innovation asymptotically (see Proposition 1) is

$$
f *=\alpha\left\{\frac{1-x^{*}}{x^{*}}\right\}=\frac{e[(1-\beta)-\gamma(1-\sigma)]-\rho \alpha(1-\beta)}{\rho(1-\beta)-e(1-\sigma)}
$$

Proof. The existence of a path converging to the equilibrium has been already shown. (Strictly, the initial values of $x, y$, and $z$ must not be too different from $x^{*}, y^{*}$, and $z^{*}$ ) Uniqueness and optimality follow from Mangasarian's Sufficiency Theorem once concavity of the maximized Hamiltonian in the state variables and the transversality conditions are established. (See Arfow and Kurz [1].) First concavity. Consider the equivalent problem 
arising if the technological parameter, $A$, is replaced by $A^{\mu}, \mu>0$. The terms in the Hamiltonian (16), involving consumption, $C$, cause no difficulty. The remaining terms are now

$$
\mathcal{H}_{1}=\left\{\frac{\Psi e\left(L-L_{p}\right)}{\mu L} A+\phi A^{\mu} L_{p}^{\alpha} K^{\beta}{ }_{R}^{\gamma}\right\} e^{-\rho t}-\lambda R
$$

The maximized value of this expression, apart from an additive constant, is

$$
\mathcal{H}_{1}^{*}=(1-\alpha-\gamma) \phi \mathrm{e}^{-\rho t} \mathrm{~A}^{\mu} \mathrm{L}_{\mathrm{P}}^{\alpha} \mathrm{K}^{\beta} \mathrm{R}^{\gamma}
$$

where

$$
L_{p}, R \cup A^{\mu /(1-\alpha-\gamma)} K^{\beta /(1-\alpha-\gamma)}
$$

so that

$$
3 C_{1}^{*} \propto A^{\mu /(1-\alpha-\gamma)} \mathrm{K}^{\beta /(1-\alpha-\gamma)}
$$

which is concave in $A$ and $K$ if

$$
\mu<\delta=1-(x-\beta-\gamma
$$

as might be expected.

Consider now the transversality condition for A.

$$
\begin{aligned}
\frac{\left(e^{-\rho t} \Psi A\right)^{\prime}}{e^{-\rho t} \Psi A} \rightarrow & -\rho+\rho-e\left(1+\frac{1-\alpha}{\alpha} x^{*}\right)+e\left(1-x^{*}\right) \\
& =-\frac{e x^{*}}{\alpha}<0
\end{aligned}
$$

Hence the present value of technology tends to zero as $t \rightarrow \infty$. Similarly; for capital since

$$
\begin{aligned}
\frac{\left(\mathrm{e}^{-\rho t} \phi \mathrm{K}\right)^{\prime}}{\mathrm{e}^{-\rho t} \phi \mathrm{K}} \rightarrow & -\rho+\rho-\beta y^{*}+\mathrm{y}^{*}-\mathrm{z}^{*} \\
& =-\frac{\rho(1-\beta)-\mathrm{e}(1-\sigma)}{[(1-\beta)-(\alpha+\gamma)(1-\sigma)]}<0
\end{aligned}
$$

using (39). The transversality condition for the stock of the depletable resource, $S$, is satisfied simply because $S$ tends to zero. 
Hence the convergent path is unique and optimal.

6. Comparative Long-Run "Statics"

\section{Proposition 3}

Asymptotically, the rate of return to innovation (as in (28)), equals the rate of exploitation of the depletable resource. For, from (46) and (48),

$$
\mathbf{r}_{I}^{*}=\operatorname{ex}^{*} / \alpha=\epsilon *
$$

This can also be seen to follow from

$$
\left(\frac{Q_{L}^{\prime}}{Q_{L}}\right)^{*}=w^{*},\left(\frac{Q_{R}^{\prime}}{Q_{R}}\right)^{*}=w^{*}+\epsilon^{*}
$$

and (22), (25), and (28) relating innovation and exploitation of the depletable resource.

The comparative growth path "statics" of Table 1 are readily obtained using (39). Note that the explicit rate of time preference, $\rho$, and the elasticity of marginal utility, $\sigma$, affect the rate of growth, w*, and do not simply shift the paths of variables by a constant percentage as in the typical neoclassical case. (See Arrow and Klirz [1], for example.) However, the impact of these two parameters is otherwise intuitive.

Consider now the effect of more productive innovation, as induced by an increase in e. As expected, the labor used for innovation and the asymptotic growth rate increase. One might then expect extra "impatience" to be reflected in an offsetting faster rate of exploitation of the depletable resource. This is not generally the case, for, from Table 1: 
Table 1

Comparative Growth Path "Statics"

Dependent Parameter

\begin{tabular}{|r|cccc|}
\cline { 3 - 5 } \multicolumn{1}{r|}{} & $\mathrm{x}^{*}$ & $\mathrm{y}^{*}$ & $\mathrm{w}^{*}$ & $\epsilon *$ \\
\hline$\rho$ & $>0$ & $>0$ & $<0$ & $>0$ \\
\hline$\sigma \quad$ (i) $w^{*}>0$ & $>0$ & $>0$ & $<0$ & $>0$ \\
(ii) $w^{*}<0$ & $<0$ & $<0$ & $>0$ & $<0$ \\
\hline e (i) $\sigma<1$ & & $>0$ & $>0$ & $<0$ \\
(ii) $\sigma>1$ & $<0$ & & & $>0$ \\
\hline$\gamma$ (i) $\sigma<1$ & $>0$ & $<0$ & $<0$ & $<0$ \\
(ii) $\sigma>1$ & $<0$ & & & $<0$ \\
\hline
\end{tabular}




\section{Proposition 4}

More productive innovation leads to greater employment in innovation, and a faster asymptotic growth rate. However, if the elasticity of marginal utility is less than one, more productive innovation also leads to a lower rate of exploitation of the depletable resource. Only when the elasticity of marginal utility is greater than one will there be a faster rate of exploitation of the depletable resource.

The explanation is as follows. The asymptotic rate of consumption interest ("impatience") indeed does increase with a faster rate, for, from (21),

$$
r_{c}^{*}=\rho+\sigma\left(\frac{C^{*}}{C}\right)^{*}=\rho+\sigma w^{*}
$$

However, so does the rate of return on holding the resource, from (54). So optimality ultimately requires

$$
\frac{r Q_{R}}{Q_{R}} \cdot=w *+\epsilon *=r_{c}^{*}=\rho+\sigma w *
$$

using (21) and (22). From (59) the result follows, given the increase in w*. Note 1: Since from Proposition $3, r_{I}^{*}=\epsilon *$, the rate of return on innovation also falls when innovation becomes more productive if and only if the elasticity of marginal utility is less than one.

Note 2: If $\sigma=1$, then $\mathrm{r}_{I}^{*}=\epsilon^{*}=\rho$, the explicit rate of time preference. Note 3: Proposition 4 might seem more striking as "Less productive innovation leads to a faster rate of exploitation of the depletable resource (and a higher rate of return on innovation) when the elasticity of marginal utility is less than one." 
Again from Table 1, consider now the impact of a higher relative share of the depletable resource (at the expense of the non-depletable resource, as explained following (2)). Such a higher share for the depletable resource is analogous to technological "regress". Not surprisingly, the asymptotic rate of growth, $w *$, declines. Then less "impatience" might be expected to lead to an offsetting decrease in the rate of exploitation of the depletable resource, to an increase in innovation, or to both. However :

Proposition 5

When the elasticity of marginal utility is less than one, a larger share of the depletable resource means less innovation and faster exploitation. Only if the elasticity of marginal utility is greater than one is there more innovation and slower exploitation.

The explanation for the effect on the rate of exploitation of the depletable resource via the decrease in the asymptotic growth rate is as explained following Proposition 4. Also, optimality ultimately requires

$$
r_{I}^{*}+w^{*}=r_{c}^{*}=\rho+\sigma w^{*}
$$

using (21) and (57). This accounts similarly for the effect on innovation. Crucial to this explanation is the fact that a reduction in the rate of increase of the wage rate inhibits innovation, as in Proposition 2.

7. The Rawlsian Criterion

Consider now the outcome as $\sigma$, the elasticity of marginal utility, tends to infinity. If $\mathrm{C}(\mathrm{t})$ is continuous

$$
\left\{\int_{0}^{\infty} c^{1-\sigma} e^{-\rho t} d t\right\}^{\frac{1}{1-\sigma}} \rightarrow \inf _{t}\{c(t)\}, \sigma \rightarrow \infty
$$


(See Rudin [10], for example.) Maximizing u[C] then becomes equivalent to the "max-min" criterion, advanced by Rawls [9]. Efficiency still requires generally a readjustment of the initial stocks of capital, technology, and the depletable resource. Indeed (32) and (35) for $x$ and $y$ derive from efficiency considerations and do not involve $\sigma$. Equation (36) becomes

$$
\frac{z^{\prime}}{z}=z-y=-\frac{k^{\prime}}{k}
$$

so that consumption, C, is constant, as expected. The system (32), (35), and (62) are necessary conditions under a Rawlsian criterion. Unique equilibrium values of $x, y$, and $z$ exist and can be obtained from (46) as $\sigma \rightarrow \infty$. A unique convergent path still exists, and it is not hard to deduce that this path corresponds to the maximum feasible constant value of consumption. Thus the limiting system has qualitatively the same behavior as the case for finite $\sigma$. (In fact, it is the presence of innovation which smooths the transition to the limit. Consider, for example, a case with no depletable resource, and zero rates of technological change, population growth and depreciation. The Rawlsian criterion implies that all output should be consumed from time zero. Hence, the capital stock is frozen at its initial level. However, for any finite $\sigma$, the equilibrium capital stock is determined by $Q_{K}=\rho$, independently of $\sigma$. Generally, then, the limit of the equilibria for finite $\sigma$ is not appropriate under the Rawlsian criterion. In terms of the phase diagram analysis of this paper, the $y^{\prime}=0$ and $z^{\prime}=0$ loci coincide in the limit.)

Consider then the balanced growth path arising under the Rawlsian criterion. Denote values under this double limit by double asterisks. From (47), 


$$
w * *=0
$$

so that output, capital, and consumption are ultimately constant. However, from (50)

$$
f * *=\gamma
$$

so that the burden of maintaining output in the face of depletion of a natural resource falls on innovation alone. Indeed (64) states that the fraction of output needed to finance innovation is precisely the share of the depletable resource. This again highlights the link between innovation and depletion of a natural resource. (Stiglitz [12] found, with no innovation, that rent on the depletable resource should equal investment. With endogenous investment, however, it is more efficient to innovate.) Consider now the limiting values of $x *, y *, z *$, and $\epsilon *$ from (46) and (48)

$$
\begin{aligned}
& \mathrm{x} * *=\alpha /(\alpha+\gamma) \\
& y * *=z * *=e /[\beta(\alpha+\gamma)] \\
& \epsilon * *=e /(\alpha+\gamma)
\end{aligned}
$$

In this case, as in general when $w^{*}=0$ (see Proposition 3 ),

$$
\epsilon * *=Q_{K}^{* *}=r_{c}^{* *}
$$

Note that the presence of innovation means that the Rawlsian asymptotic rate of interest, $r_{c}^{* *}$ is independent of the initial conditions. Also, using (47),

$$
r_{c}^{* *}<\rho \text { if } w^{*}<0
$$

where $w^{*}$ is the asymptotic growth rate for finite $\sigma$, the elasticity of marginal utility.

\section{Proposition 6}

With a Rawlsian criterion, it is ultimately optimal to use the rent from the depletable resource to finance innovation, and to hold the capital stock 
constant. The rate of exploitation of the depletable resource should finally equal the asymptotic rate of interest.

Propositions 4 and 5 might be interpreted as mildly strengthening the "reasonableness" of a Rawlsian criterion. Note generally that the non-paradoxical case with the elasticity of marginal utility greater than one is inconsistent with monopoly control of the depletable resource (or at any stage). A government then simply could not adopt such a dynamic utility functional in the face of the ofl cartel, for example.

\section{Conclusions}

This paper demonstrates that there is basic symmetry between costly innovation and natural resource depletion. These phenomena are shown to affect the asymptotic growth rate, whereas capital accumulation does not. In the limit, too, the rate of return on innovation equals the rate of exploitation of the natural resource. Furthermore, under the Rawlsian criterion the rent from the depletable resource should eventually be used to finance innovation, while no investment should occur. However, the interaction of innovation and resource depletion can sometimes yield unexpected results. It is possible, for example, that more efficient innovation leads to a slower rate of exploitation of the resource. Similarly, a larger output share for the depletable resource can lead to less innovation and a faster rate of exploitation.

It would be interesting to generalize the function used to describe innovation and the production function. What then is the appropriate rate of return on innovation and how does it relate to the marginal product of capital? What is the general condition under which innovation dominates investment? To treat this general case requires a "tight" set of sufficient conditions to ensure "global asymptotic stability" with costly innovation and depletable natural resources. 
References

[1] Arrow, K and Kurz, M. Public Investment, the Rate of Return and Optimal Fiscal Policy (Johns Hopkins, Baltimore, 1970).

[2] Brock, W. and Scheinkman, J. "Global Asymptotic Stability of Optimal Control Systems with Applications to the Theory of Economic Growth," Journal of Economic Theory, 12 (1976), 164-190.

[3] Conlisk, J. "A Neoclassical Growth Model with Endogenously Positioned Technical Change Frontier," Economic Journal, 79 (1969), 348-362.

[4] Dasgupta, P. and Heal, G。 "The Optimal Depletion of Exhaustible Resources," Review of Economic Studies, Symposium on the Economics of Exhaustible Resources $(1974), 3-28$.

[5] Hotelling, H. "The Economics of Exhaustible Resources," Journal of Political Economy (1931).

[6] Kennedy, C. "Induced Bias in Innovation and the Theory of Distribution," Economic Journal, 74 (1964), 541-547.

[7] Meadows, D. H. et al. The Limits to Growth (Universe Books, New York, 1972)。

[8] Phelps, E. Golden Rules of Economic Growth (W. W. Norton, New York, 1966).

[9] Rawls, J. A Theory of Justice (Harvard University Press, Cambridge, 1971)。

[10] Rudin, W. Real and Complex Analysis (McGraw-Hill, New York, 1974)。

[11] Solow, R. M。 "Intergenerational Equity and Exhaustible Resources," Review of Economic Studies, Symposium on the Economics of Exhaustible Resources (1974), 29-45.

[12] Stiglitz, J。 "Growth with Exhaustible Natural Resources," Review of Economic Studies, Symposium on the Economics of Exhaustible Resources (1974), 123-137. 